\title{
ON DEFORMABLE MINIMAL HYPERSURFACES IN SPACE FORMS
}

\author{
ANDREAS SAVAS-HALILAJ
}

\begin{abstract}
The aim of this paper is to complete the local classification of minimal hypersurfaces with vanishing Gauss-Kronecker curvature in a 4-dimensional space form. Moreover, we give a classification of complete minimal hypersurfaces with vanishing Gauss-Kronecker curvature and scalar curvature bounded from below.
\end{abstract}

\section{INTRODUCTION}

A classical result stated by R. Beez [2], but completely proved by W. Killing [15], says that a hypersurface in the Euclidean space $\mathbb{R}^{n+1}$ is locally rigid if its type number, i.e., the rank of its Gauss map, is at least 3. Hence, the interesting case is that of hypersurfaces with type number less than 3.

Inspired by works of Sbrana 22] and Cartan [5], M. Dajczer and D. Gromoll 9 proved that every hypersurface with constant type number in $\mathbb{R}^{n+1}$ can be described in terms of its Gauss map. This kind of description is called the "Gauss parametrization". However, the Gauss parametrization provides a description of the hypersurface at the points where the type number is constant and there is no discussion on the manner in which hypersurfaces with different type numbers can be produced or joined together.

In this paper we consider minimal hypersurfaces with type number less than 3 in $\mathbb{Q}_{c}^{4}$. Our aim is to provide a local parametrization of such hypersurfaces around the totally geodesic points. In the 3 -dimensional case the assumption that the type number is less than 3 is equivalent to the assumption that the GaussKronecker curvature vanishes. It turns out that hypersurfaces with constant type number 2 are highly deformable since they allow locally an one-parameter family of isometric deformations, the so called associated family. We shall show that such hypersurfaces are either totally geodesic or arise, at least locally, as normal bundles of certain branched minimal surfaces. More precisely, we shall show the following result.

Theorem 1.1. Let $M^{3}$ be an oriented Riemannian manifold of dimension 3 and $f: M^{3} \rightarrow \mathbb{Q}_{c}^{4}$ a minimal isometric immersion with vanishing Gauss-Kronecker curvature. Then, $f\left(M^{3}\right)$ is either totally geodesic in $\mathbb{Q}_{c}^{4}$ or, at least locally, coincide with the polar map of a branched minimal surface.

For the definition of the polar map over branched minimal surfaces see Section 4 .

2000 Mathematics Subject Classification. Primary 53C40; Secondary 53C42.

Key words and phrases. Harmonic map, Gauss map, branch point, minimal hypersurface, Gauss-Kronecker curvature, real analytic set. 
Dajczer and Gromoll [9] showed that in order to solve the classification problem for the class of complete minimal hypersurfaces with type number less than 3 , one has to understand the nature of 3-dimensional complete minimal hypersurfaces with vanishing Gauss-Kronecker curvature in $\mathbb{Q}_{c}^{4}$.

In [1] S.C. de Almeida and F.G.B. Brito initiated the study of compact minimal hypersurfaces in $\mathbb{S}^{4}$ with vanishing Gauss-Kronecker curvature and gave a classification under the assumption that the second fundamental form of the hypersurface nowhere vanishes. J. Ramanathan in [20] removed the assumption on the second fundamental form and proved that such hypersurfaces arise as normal bundles over branched minimal surfaces in $\mathbb{S}^{4}$, unless they are totally geodesic. We point out, that Theorem 1.1 generalizes Ramanathan's result, since the same conclusion holds without the assumption of compactness.

Complete minimal hypersurfaces with zero Gauss-Kronecker curvature in $\mathbb{Q}_{c}^{4}$ was investigated by $\mathrm{T}$. Hasanis et al. in [11, 12, 13]. In particular, a classification of such hypersurfaces was given, under the assumptions that the scalar curvature is bounded from below and that the second fundamental form of $f$ is nowhere zero. In the following theorem, we generalize results in [11, 12, 13, 20].

Theorem 1.2. Let $M^{3}$ be an oriented, 3-dimensional, complete Riemannian manifold with scalar curvature bounded from below. If $f: M^{3} \rightarrow \mathbb{Q}_{c}^{4}$ is a minimal isometric immersion with Gauss-Kronecker curvature identically zero, then either $f$ is totally geodesic, or

a) $c=0$ and $f\left(M^{3}\right)$ splits as a Euclidean product $M^{2} \times \mathbb{R}$, where $M^{2}$ is a complete minimal surface in $\mathbb{R}^{3}$,

b) $c=1$ and $f\left(M^{3}\right)$ is the image of the polar map associated with a branched superminimal immersion $g: M^{2} \rightarrow \mathbb{S}^{4}$,

c) $c=-1$ and $f\left(M^{3}\right)$ is the hyperbolic cylinder over a complete minimal surface $M^{2}$ in $Q^{3}$, where $Q^{3}$ is a horosphere or an equidistant hypersurface in $\mathbb{H}^{4}$.

The paper is organized as follows. In Section 2 we introduce the necessary background about harmonic maps. In Section 3 we review some basic facts about the behavior of the Gauss map of branched minimal surfaces. In Section 4 we furnish a method to produce minimal hypersurfaces in $\mathbb{Q}_{c}^{4}$ with zero Gauss-Kronecker curvature. Section 5 is devoted to the study of the set of totally geodesic points of such hypersurfaces. Finally, in Section 6 we the prove Theorem 1.1 and Theorem 1.2.

\section{HARMONIC MAPS}

Let $M^{m}$ and $N^{n}$ be two differentiable manifolds, of dimensions $m$ and $n$, equipped with Riemannian metrics $\langle,\rangle_{M}$ and $\langle,\rangle_{N}$, respectively. Denote by $\nabla^{M}$ and $\nabla^{N}$ the corresponding Levi-Civita connections. If $F: M^{m} \rightarrow N^{n}$ is a smooth map, we define the energy integral of $F$ over a compact domain $\Omega$ of $M^{m}$ by

$$
E(F, \Omega):=\frac{1}{2} \int_{\Omega}\|d F\|^{2} d M
$$

where $d M$ is the volume element of $M^{m}$ and $\|d F\|(p)$ is the Hilbert-Schmidt norm of the differential $d F: T M^{m} \rightarrow T N^{n}$ of $F$ at a point $p$ of $\Omega$. A smooth map $F$ is said to be harmonic if it is a critical point of the energy integral $E(\cdot, \Omega)$, with respect to variations supported in $\Omega$. 
The Hessian $\nabla^{2} F: T M^{m} \times T M^{m} \rightarrow F^{*}\left(T N^{n}\right)$ of $F$ is defined by

$$
\nabla^{2} F(X, Y):=\nabla_{X}^{F} d F(Y)-d F\left(\nabla_{X}^{M} Y\right), \quad X, Y \in T M^{m},
$$

where $F^{*}\left(T N^{n}\right)$ stands for the induced vector bundle by $F$ and $\nabla^{F}$ for the induced Levi-Civita connection of $F^{*}\left(T N^{n}\right)$. Taking the Euler-Lagrange equations, it follows that $F$ is harmonic if and only if the trace with respect to $\langle,\rangle_{M}$ of the Hessian $\nabla^{2} F$ vanishes identically. In local coordinates $(U, \phi)$ on $M^{m}$ and $(V, \psi)$ on $N^{n}$, the harmonicity of $F$ is expressed by the following system of elliptic differential equations

$$
\Delta f^{\alpha}+\sum_{\alpha, \beta=1}^{n} \Gamma_{\beta \gamma}^{\alpha} \circ F\left\langle\nabla^{M} f^{\beta}, \nabla^{M} f^{\gamma}\right\rangle_{M}=0, \quad \alpha \in\{1,2, \cdots, n\},
$$

where $\Delta$ is the Laplace operator on $M^{m}, f^{\alpha}$ the components of $F$ and $\Gamma_{\beta \gamma}^{\alpha}$ the Christoffel symbols of $N^{n}$ with respect to the coordinate system $(V, \psi)$.

The notion of harmonic map can be carried out successfully even in the case of semi Riemannian manifolds without any changes. However, in this article we shall need only the case of harmonic maps $F: M^{2} \rightarrow N_{1}^{n}$, where $N_{1}^{n}$ is a semi Riemannian manifold of signature $(1, n-1)$. We point that in this case the system of differential equations (2.1) is still elliptic.

An isometric immersion between Riemannian manifolds is harmonic if and only if it is minimal. Moreover, any harmonic map between real analytic manifolds with real analytic Riemannian metrics is also real analytic (cf., [6, p. 117]). According to the so called Unique Continuation Property, states that two harmonic maps $F_{1}, F_{2}: M^{m} \rightarrow N^{n}$ which coincide in an open subset $\Omega$ of $M^{n}$ should coincide in all of $M^{m}$ (cf., 21, Theorem 1, p. 213]).

Let assume that $\mathcal{K}$ is a compact set in some open ball $B_{R}(0) \subset \mathbb{R}^{n}$ with radius $R$ centered at the origin of $\mathbb{R}^{n}$. Then, the relative 2 -capacity of $\mathcal{K}$ is defined to be

$\operatorname{cap}_{2, R}(\mathcal{K}):=\inf \left\{\int_{\mathbb{R}^{n}}|\nabla \varphi|^{2} d \mathbb{R}^{n}: \varphi \in C_{c}^{\infty}\left(B_{R}(0)\right)\right.$ and $\varphi(x) \geq 1$, for all $\left.x \in \mathcal{K}\right\}$,

where $C_{c}^{\infty}\left(B_{R}(0)\right)$ is the space of smooth functions $\varphi: B_{R}(0) \rightarrow \mathbb{R}$ with compact support. The relative 2 -capacity measures how "small" must be a set $\mathcal{K}$ in order a harmonic map to be extendable over it. For $n \geq 3$, we set $\operatorname{cap}_{2}(\mathcal{K})=\operatorname{cap}_{2, \infty}(\mathcal{K})$. If $\mathcal{C}$ is an arbitrary subset of $\mathbb{R}^{n}$ we say that $\operatorname{cap}_{2}(\mathcal{C})=0$ if and only if $\operatorname{cap}_{2}(\mathcal{K})=0$, for any compact subset $\mathcal{K}$ of $\mathcal{C}$. In particular, if $M^{m}$ is an $m$-dimensional differentiable manifold and $\mathcal{C}$ a subset of $M^{m}$, then we say that $\operatorname{cap}_{2}(\mathcal{C})=0$ if and only if for any coordinate chart $(U, \phi)$ on $M^{m}$ it holds $\operatorname{cap}_{2}(\phi(\mathcal{C} \cap U))=0$. Finally, there is a close relation between the 2-capacity and the $(m-2)$-Hausdorff measure $\mathcal{H}^{m-2}$. In fact, the following result is well known (cf., [8, pp. 154-158]).

Theorem 2.1. Let $\mathcal{K}$ be a compact set. Then, the following assertions are valid:

a) If $\mathcal{H}^{m-2}(\mathcal{K})<\infty$, then $\operatorname{cap}_{2}(\mathcal{K})=0$.

b) If $\operatorname{cap}_{2}(\mathcal{K})=0$, then $\mathcal{H}^{s}(\mathcal{K})=0$ for any $s>m-2$.

For example, if $\mathcal{C}$ is a picewise smooth curve in $\mathbb{R}^{3}$, then $\mathcal{H}^{1}(\mathcal{C})=1$ and consequently $\operatorname{cap}_{2}(\mathcal{C})=0$. M. Meier in [17] provides a criterion about the removability of singularities for harmonic maps between Riemannian manifolds. 
Theorem 2.2. Let $M^{m}$ be a $m$-dimensional Riemannian manifold, $\mathcal{C}$ a relatively closed subset of $M^{m}$ with $\operatorname{cap}_{2}(\mathcal{C})=0$ and $N^{n}$ a n-dimensional Riemannian manifold. If $F: M^{m} \rightarrow N^{n}$ is a map satisfying the following properties:

a) $F$ is continuous on $M^{m}$,

b) $F$ is harmonic on $M^{m}-\mathcal{C}$.

then, $F$ extends analytically to a harmonic map in all of $M^{m}$.

\section{The GAUSs MAP OF BRANCHED SURFACES}

In this section we review some basic facts about the Gauss map of spacelike branched minimal surfaces in semi Riemannian manifolds.

Denote by $\mathbb{R}_{s}^{n}$ the a $n$-dimensional vector space $\mathbb{R}^{n}$ endowed with the semi Riemannian metric $\langle,\rangle_{s}$ given by

$$
\langle v, w\rangle_{s}:=(-1)^{s} v_{1} w_{1}+v_{2} w_{2}+\cdots+v_{n} w_{n}, \quad s \in\{0,1\},
$$

where $v=\left(v_{1}, v_{2}, \cdots, v_{n}\right)$ and $w=\left(w_{1}, w_{2}, \cdots, w_{n}\right)$. The Grassmannian space of the 2-dimensional planes in $\mathbb{R}_{s}^{n}$ is denoted by $\mathbb{G}_{2}\left(\mathbb{R}_{s}^{n}\right)$ and admits a differentiable structure of dimension $2(n-2)$. A 2 -plane $\Pi$ in $\mathbb{R}_{s}^{n}$ is said to be spacelike, if the restriction to $\Pi$ of the inner product $\langle,\rangle_{s}$ is positive definite. Let $\mathbb{G}_{2}^{+}\left(\mathbb{R}_{s}^{n}\right)$ the space of all oriented spacelike 2-planes in $\mathbb{R}_{s}^{n}$. Consider a 2-dimensional oriented Riemannian manifold $\left(M^{2},\langle\rangle,\right)$ endowed with a fixed complex structure as a Riemann surface, a $n$-dimensional semi Riemannian manifold $\left(N_{s}^{n},\langle,\rangle_{s}\right)$ equipped with a semi Riemannian metric of signature $(s, n-s), s \in\{0,1\}$. Assume that $N_{s}^{n}$ can be isometrically immersed into a Minkowski space $\mathbb{R}_{s}^{n+l}$. The latest assumption is not essential for $s=0$ since, according to a well known result of J. Nash, any Riemannian manifold can be isometrically immersed into a Euclidean space.

We say that $g: M^{2} \rightarrow N_{s}^{n} \subset \mathbb{R}_{s}^{n+l}$ is a (weakly) conformal map, if

$$
g^{*}\langle,\rangle_{s}=E\langle,\rangle
$$

where $E: M^{2} \rightarrow \mathbb{R}$ is a smooth non negative function, the so called conformal factor. On the open set $M_{*}^{2}$ of its regular points, $g$ is a spacelike immersion and its Gauss map $\xi: M_{*}^{2} \rightarrow \mathbb{G}_{2}^{+}\left(\mathbb{R}_{s}^{n+l}\right)$ is defined by

$$
\xi(x):=d g\left(T_{x} M_{*}^{2}\right), \quad x \in M^{2} .
$$

If $x_{0}$ is a critical point of $g$, then the tangent plane of $g$ at $x_{0}$ does not exists in general. A critical point $x_{0}$ of $g$ is called branch point of order $m, m \in \mathbb{N}$, if there are coordinate charts $(U, \varphi)$ centered at $x_{0}$ and $(V, \psi)$ around $g\left(x_{0}\right)$, such that the representation map $\widetilde{g}: \varphi(U) \rightarrow \mathbb{C} \times \mathbb{R}^{n-2}, \widetilde{g}:=\psi \circ g \circ \varphi^{-1}$, has the form

$$
\widetilde{g}(z)=\left(z^{m+1}+h_{1}(z)+\sqrt{-1} h_{3}(z), h(z)\right), \quad z \in \varphi(U),
$$

where $h_{1}(z), h_{2}(z), h(z) \in O\left(|z|^{m+2}\right)$. In particular, $g$ is called branched surface if each critical point is a branch point. According to results due to R.D. Gulliver et al. [18, Lemma 1.3 and Lemma 3.1], the branch points are isolated and the Gauss map can be continuously extended over the branch points. It turns out that the Gauss map of a conformal harmonic map can be smoothly extended over the branch points. More precisely, the following theorem due to J.-H. Eschenburg and R. Tribuzy [7] provides a characterization of the critical points of a conformal harmonic map. 
Theorem 3.1. Let $M^{2}$ be a Riemann surface and $N_{s}^{n}$ a semi Riemannian manifold of signature $(s, n-s), s \in\{0,1\}$, which can be isometrically immersed into a Minkowski space $\mathbb{R}_{s}^{n+l}$. Assume that $g: M^{2} \rightarrow N_{s}^{n} \subset \mathbb{R}_{s}^{n+l}$ is a conformal harmonic map. Then,

a) the critical points of $g$ are branch points.

b) The Gauss map of $g$ extends smoothly over the branch points.

c) If $x_{0} \in M^{2}$ is a branch point of order $m \geq 1$, then for any holomorphic chart $(U, z), z: U \rightarrow \mathbb{C}$, centered at $x_{0}$, we have

$$
E(z)=|z|^{2 m} E_{0}(z) \text {, }
$$

where $E_{0}$ is a smooth positive function on $U$.

Remark 3.2. Although the proof in [7] was given for surfaces lying in a Riemannian manifold, the same technique can be applied also in the case of spacelike surfaces in a semi Riemannian manifold $N_{1}^{n}$ of signature $(1, n-1)$, which can be immersed isometrically in $\mathbb{R}_{1}^{n+l}$.

\section{Gauss parametrization}

In this section we shall describe a method of constructing minimal hypersurfaces with vanishing Gauss-Kronecker curvature in a space form $\mathbb{Q}_{c}^{4}$ and will illustrate the results with some examples.

Consider a branched minimal surface $g: M^{2} \rightarrow \mathbb{S}^{3} \subset \mathbb{R}^{4}$, where $M^{2}$ is an oriented manifold of dimension 2. Denote by $\langle,\rangle_{g}$ the induced by $g$ metric tensor on $M^{2}$. On the open set $M_{*}^{2}$ of its regular points, $g$ is an immersion and $\langle,\rangle_{g}$ is a Riemannian metric. According to Theorem 3.1, the normal bundle

$$
\mathcal{N}(g):=\left\{(x, w) \in M^{2} \times \mathbb{R}^{4}: w \perp \mathbb{R} \cdot g+d g\left(T_{x} M^{2}\right)\right\},
$$

is a well defined smooth manifold of dimension 3. Let $\gamma: M^{2} \rightarrow \mathbb{R}$ be a smooth function and denote by $\nabla \gamma$ its gradient, by $\nabla^{2} \gamma$ its Hessian and by $\Delta \gamma$ its Laplacian with respect to $\langle,\rangle_{g}$ on $M_{*}^{2}$. Assume further that $d g(\nabla \gamma)$ can be smoothly extended over the branch points of $g$. Then, the map $\Psi_{\gamma, g}: \mathcal{N}(g) \rightarrow \mathbb{R}^{4}$ given by

$$
\Psi_{\gamma, g}(x, w):=\gamma(x) g(x)+d g(\nabla \gamma)(x)+w,
$$

is well defined and smooth. The map $\Psi_{\gamma, g}$, when restricted on the normal bundle of $g: M_{*}^{2} \rightarrow \mathbb{S}^{3}$, coincides with the polar map invented by Dajczer and Gromoll in [9, Section 1]. Since our considerations are of local nature, we shall replace $M^{2}$ with the open unit disc

$$
B:=\{z \in \mathbb{C}:|z|<1\} .
$$

From now on denote by $B_{*}:=B-\{0\}$ the punctured open unit disc.

Proposition 4.1. Let $g: B \rightarrow \mathbb{S}^{3} \subset \mathbb{R}^{4}$ be a branched minimal surface, having $z=0$ as branch point of order $m$ and being everywhere else regular. Assume that $\gamma: B \rightarrow \mathbb{R}$ is a smooth function, satisfying on $B_{*}$ the differential equation

$$
\Delta \gamma+2 \gamma=0
$$

Then,

a) the polar map is well defined and smooth if and only if $d g(\nabla \gamma)$ can be smoothly extended over the branch point. 
b) Let $A_{w}$ be the shape operator of $g$ with respect to the normal $w$. The map $\Psi_{\gamma, g}$ is regular at $(z, w), z \neq 0$, if and only if the self adjoint operator $P: T_{z} B \rightarrow T_{z} B$ given by

$$
P:=\nabla^{2} \gamma+\gamma I-A_{w}
$$

is non-singular. Moreover, $\Psi_{\gamma, g}$ is regular at $(0, w)$ if and only if there is a negative number $l$ such that

$$
\lim _{z \rightarrow 0}|z|^{2 m} \operatorname{det} P=l \text {. }
$$

c) On the open set of its regular points, the polar map is minimal with vanishing Gauss-Kronecker curvature.

Proof. Since $z=0$ is a branch point, according to Theorem 3.1, there is a positive smooth function $E_{0}$ on $B$ such that

$$
\langle,\rangle_{g}=|z|^{2 m} E_{0}\left(d x^{2}+d y^{2}\right) \text {. }
$$

Denote by $\eta$ a unit normal vector field along $g$. Parametrize $\mathcal{N}(g)$ by $B \times \mathbb{R}$ via the map $(z, t) \rightarrow(z, t \eta(z))$. Then, for any $(z, t) \in B_{*} \times \mathbb{R}$,

$$
\Psi_{\gamma, g}(z, t)=\gamma(z) g(z)+d g(\nabla \gamma)(z)+t \eta(z) .
$$

a) It is obvious that the polar map is well defined and smooth if and only if $d g(\nabla \gamma)$ can be smoothly extended over the branch point.

$b$ ) On an arbitrary point $(z, t), z \neq 0$, we have

$$
d \Psi_{\gamma, g}\left(\partial_{t}\right)=\eta
$$

and, for any $X \in T_{z} B$,

$$
d \Psi_{\gamma, g}(X)=d g(P X)+\omega(X) \eta,
$$

where $\omega$ stands for the dual form of the vector $A_{\eta} \nabla \gamma$. The metric tensor $\langle,\rangle_{\Psi}$ induced on $B_{*} \times \mathbb{R}$ by $\Psi_{\gamma, g}$ is given by the formula

$$
\langle,\rangle_{\Psi}=\left\langle P^{2},\right\rangle_{g}+(d t+\omega) \otimes(d t+\omega) \text {. }
$$

Since $\gamma$ satisfies the differential equation $\Delta \gamma+2 \gamma=0$, we deduce that

$$
\begin{aligned}
\langle,\rangle_{\Psi} & =-\operatorname{det} P\langle,\rangle_{g}+(d t+\omega) \otimes(d t+\omega) \\
& =-|z|^{2 m} E_{0} \operatorname{det} P\left(d x^{2}+d y^{2}\right)+(d t+\omega) \otimes(d t+\omega) .
\end{aligned}
$$

Hence, the polar map is regular at $(z, t), z \neq 0$, if and only if $\operatorname{det} P \neq 0$. Since,

$$
\omega(X)=\left\langle A_{\eta} \nabla \gamma, X\right\rangle_{g}=-\langle d \eta(X), d g(\nabla \gamma)\rangle
$$

it follows that $\omega$ is well defined and smooth over the branch point. Moreover, $\Psi_{\gamma, g}$ is regular at $(0, t)$ if and only if there exists a negative real number $l$, such that

$$
\lim _{z \rightarrow 0}|z|^{2 m} \operatorname{det} P=l \text {. }
$$

c) The unit vector field $\xi$ given by $\xi(z, t):=g(z)$ is smooth and normal along $\Psi_{\gamma, g}$. Suppose now that $\left(0, t_{0}\right)$ is a regular point of $\Psi_{\gamma, g}$. Then, there exists a neighborhood $U$ around $\left(0, t_{0}\right)$ where $\Psi_{\gamma, g}$ is regular. Since,

$$
\lim _{z \rightarrow 0}|z|^{2 m} \operatorname{det} P=l<0,
$$

there exists a continuous positive function $\Phi: U \rightarrow \mathbb{R}$, such that

$$
-|z|^{2 m} \operatorname{det} P=\Phi(z, t), \quad(z, t) \in U \text {. }
$$


By (4.1), it follows that the principal curvatures of $\Psi_{\gamma, g}$ on $U$ are given by

$$
k_{1}(z, t)=-k_{3}(z, t)=\frac{|z|^{m}}{\sqrt{\Phi(z, t)}}, \quad k_{2}(z, t)=0 .
$$

and the proof of the theorem is complete.

Example 4.2. Let $G: B \rightarrow \mathbb{S}^{3} \subset \mathbb{R}^{4}$ be a minimal immersion, $\langle,\rangle_{G}$ the induced Riemannian metric on $B$ by $G$ and $A_{G}$ the shape operator of $G$ with respect to a unit normal vector field $\eta$ along $G$. The map $g: B \rightarrow \mathbb{S}^{3}$ which assigns to each point of $B$ the endpoint of $\eta$, when this is translated to the origin of $\mathbb{R}^{4}$, coincide with the Gauss map of $G$ and is harmonic. Furthermore, the induced metric tensor $\langle$,$\rangle by g$ on $B$ is given by the formula

$$
\langle,\rangle=-\operatorname{det} A_{G}\langle,\rangle_{G} .
$$

According to [16, Lemma 1.4, p. 338], the zeroes of $\operatorname{det} A$ are isolated and of even order, unless $G$ is totally geodesic and $\operatorname{det} A$ vanishes identically. Suppose that $z=0$ is an isolated totally geodesic point of $G$. Then, there exists a smooth positive function $\Lambda$ such that

$$
-\operatorname{det} A_{G}=|z|^{2 m} \Lambda^{2}, \quad z \in B .
$$

Thus, $z=0$ is a branch point of $g$ with order $m$. Denote by $\nabla$ the Levi-Civita connection of $\langle$,$\rangle and by A$ the shape operator of $g$ on $B_{*}$. From the Weingarten formula it follows that $A_{G} A=I$. Let $\alpha$ be a constant vector of $\mathbb{R}^{4}$ and $\gamma, \sigma: B \rightarrow \mathbb{R}$ the functions given by the formulas $\gamma:=\langle g, \alpha\rangle$ and $\sigma:=\langle G, \alpha\rangle$, respectively. Then, for any $z \in B_{*}$,

$$
d g(\nabla \gamma)=\alpha^{G} \text { and } \nabla^{2} \gamma=\sigma A-\gamma I,
$$

where $\alpha^{G}$ is the tangential component of $\alpha$ along $G$. Hence, $d g(\nabla \gamma)$ is well defined and smooth over the branch point $z=0$. Moreover, $\gamma$ satisfies the differential equation $\Delta \gamma+2 \gamma=0$ on $B_{*}$, where $\Delta$ stands for the Laplacian with respect to $\langle$,$\rangle . Consider now the polar map \Psi_{\gamma, g}: B \times \mathbb{R} \rightarrow \mathbb{R}^{4}$,

$$
\Psi_{\gamma, g}(z, t)=\gamma(z) g(z)+d g(\nabla \gamma)(z)+t G(z) .
$$

For any $z \in B_{*}$, we have

$$
\operatorname{det} P=\frac{(\sigma-t)^{2}}{\operatorname{det} A_{G}}=-\frac{(\sigma-t)^{2}}{|z|^{m} \Lambda^{2}} .
$$

Consequently, the polar map fails to be regular at points $(z, t)$ with $t=\sigma(z)$. On the open set of its regular points, $\Psi_{\gamma, g}$ has principal curvatures

$$
k_{1}(z, t)=-k_{3}(z, t)=\frac{|z|^{m} \Lambda(z)}{|\sigma(z)-t|}, \quad k_{2}(z, t)=0 .
$$

Notice that points of the form $(0, t), t \neq \sigma(z)$, corresponds to totally geodesic points of the polar map.

Example 4.3. Assume that $g: B \subset \mathbb{S}^{2} \rightarrow \mathbb{S}^{3}$ is totally geodesic immersion and $\gamma: B \rightarrow \mathbb{R}$ a smooth function satisfying $\Delta \gamma+2 \gamma=0$. Then, the polar map $\Psi_{\gamma, g}$ is a cylinder over a minimal surface lying in $\mathbb{R}^{3}$. Conversely, the cylinder over a complete minimal surface in $\mathbb{R}^{3}$, gives rise to a complete minimal hypersurface with vanishing Gauss-Kronecker curvature in $\mathbb{R}^{4}$. The cylinders are the only known examples of complete minimal hypersurfaces with Gauss-Kronecker identically zero in $\mathbb{R}^{4}$. 
Assume that $M^{2}$ is an oriented manifold of dimension 2 and $g: M^{2} \rightarrow \mathbb{S}^{4} \subset \mathbb{R}^{5}$ a branched minimal surface. Then, the unit normal bundle

$$
\mathcal{N}_{1}(g):=\left\{(x, w) \in M^{2} \times \mathbb{R}^{5}:|w|=1, w \perp \mathbb{R} \cdot g+d g\left(T_{x} M^{2}\right)\right\}
$$

is a well defined smooth manifold of dimension 3. The map $\Psi_{g}: \mathcal{N}_{1}(g) \rightarrow \mathbb{S}^{4}$ given by

$$
\Psi_{g}(x, w):=w,
$$

is called the polar map over $g$.

Following the notation of Proposition 4.1, we obtain the following result.

Proposition 4.4. Let $g: B \rightarrow \mathbb{S}^{4} \subset \mathbb{R}^{5}$ be a branched minimal surface, having $z=0$ as a branch point of order $m$ and being everywhere else regular. Then,

a) a point $(z, w), z \neq 0$, is a regular point of $\Psi_{g}$ if and only if the shape operator $A_{w}$ of $g$, with respect to the normal $w$, is non-singular. Moreover, $\Psi_{g}$ is regular at a point of the form $(0, w)$, if and only if there exists a negative number $l$ such that

$$
\lim _{z \rightarrow 0}|z|^{2 m} \operatorname{det} A_{w}=l .
$$

b) On the open set of its regular points, the polar map is minimal with vanishing Gauss-Kronecker curvature.

Proof. Since $z=0$ is a branch point of $g$, the metric tensor $\langle,\rangle_{g}$ can be expressed as

$$
\langle,\rangle_{g}=|z|^{2 m} E_{0}\left(d x^{2}+d y^{2}\right)
$$

where $E_{0}$ is a positive smooth function on $B$. Denote by $\eta_{3}, \eta_{4}$ an orthonormal frame field along $g$ and parametrize $\mathcal{N}_{1}(g)$ by $B \times \mathbb{S}^{1}$ via the map $(z, t) \rightarrow$ $\left(z, \cos t \eta_{3}(z)+\sin t \eta_{4}(z)\right)$. Then, $\Psi_{g}$ can be represented as

$$
\Psi_{g}(z, t)=\cos t \eta_{3}(z)+\sin t \eta_{4}(z) .
$$

Notice that the connection form

$$
\omega_{34}(X):=\left\langle d \eta_{3}(X), \eta_{4}\right\rangle
$$

is well defined and smooth even at the branch point of $g$.

a) Let $(z, t), z \neq 0$, be a point of $B \times \mathbb{S}^{1}$. Then,

$$
d \Psi_{g}\left(\partial_{t}\right)=-\sin t \eta_{3}+\cos t \eta_{4}
$$

and, for any $X \in T_{z} B$,

$$
d \Psi_{g}(X)=-d g\left(A_{w} X\right)+\omega_{34}(X) d \Psi_{g}\left(\partial_{t}\right) .
$$

The induced by $\Psi_{g}$ metric tensor $\langle,\rangle_{\Psi}$ on $B_{*} \times \mathbb{S}^{1}$ is given by the formula

$$
\langle,\rangle_{\Psi}=-|z|^{2 m} E_{0} \operatorname{det} A_{w}\left(d x^{2}+d y^{2}\right)+\left(d t+\omega_{34}\right) \otimes\left(d t+\omega_{34}\right) .
$$

Hence, $(z, t), z \neq 0$, is a regular point of $\Psi_{g}$ if and only if the shape operator $A_{w}$ of $g$ with respect to the normal $w$ is non-singular. Moreover, $(0, t)$ is a regular point of $\Psi_{g}$ if and only if there is a negative real number $l$ such that

$$
\lim _{z \rightarrow 0}|z|^{2 m} \operatorname{det} A_{w}=l \text {. }
$$


b) The unit vector field $\xi(z, t):=g(z)$ is smooth and normal along $\Psi_{g}$. Assume that $\left(0, t_{0}\right)$ is a regular point of the polar map. Then, there exists a neighborhood $U$ around $\left(0, t_{0}\right)$, where $\Psi_{g}$ is regular. Since,

$$
\lim _{z \rightarrow 0}|z|^{2 m} \operatorname{det} A_{w}=l<0,
$$

there exists a continuous positive function $\Phi: U \rightarrow \mathbb{R}$, such that

$$
-|z|^{2 m} \operatorname{det} A_{w}=\Phi(z, t), \quad(z, t) \in U .
$$

Then, by (4.2), we deduce that $\Psi_{g}$ has principal curvatures

$$
k_{1}(z, t)=-k_{3}(z, t)=\frac{|z|^{m}}{\sqrt{\Phi(z, t)}}, \quad k_{2}(z, t)=0
$$

and the proof is complete.

Example 4.5. Let $G: B \rightarrow \mathbb{S}^{3} \subset \mathbb{S}^{4}$ be a minimal immersion having $z=0$ as an isolated totally geodesic point of order $2 \mathrm{~m}$. Then, its Gauss map $g: M^{2} \rightarrow \mathbb{S}^{3}$ is a branched minimal surface, having $z=0$ branch point of order $m$. If $A_{G}$ is the shape operator of $G$, then there exists a positive smooth function $\Lambda$ such that

$$
-\operatorname{det} A_{G}=|z|^{2 m} \Lambda^{2} \text {. }
$$

Consider $\mathbb{S}^{3}$ as a totally geodesic hypersurface of $\mathbb{S}^{4}$ and let $\alpha$ be the unit normal vector of $\mathbb{S}^{3}$ in $\mathbb{S}^{4}$. The polar map $\Psi_{g}: B \times \mathbb{S}^{1} \rightarrow \mathbb{S}^{4}$ has the form

$$
\Psi_{g}(z, t)=\cos t \alpha+\sin t G(z) .
$$

The determinant of the shape operator $A_{w}$ of $g$, with respect to a unit normal $w=\cos t \alpha+\sin t G$, is equal to

$$
\operatorname{det} A_{w}=\frac{\cos ^{2} t}{\operatorname{det} A_{G}}=-\frac{\cos ^{2} t}{|z|^{2 m} \Lambda^{2}} .
$$

Thus, $\Psi_{g}$ fails to be an immersion at points $(z, t)$ with $t=(2 n+1) \pi / 2, n \in \mathbb{Z}$. Furthermore, on the open set of its regular points, $\Psi_{g}$ has principal curvatures

$$
k_{1}(z, t)=-k_{3}(z, t)=\frac{|z|^{m} \Lambda(z)}{|\cos t|}, \quad k_{2}(z, t)=0 .
$$

Notice that points of the form $(0, t), t \neq(2 n+1) \pi / 2, n \in \mathbb{Z}$, corresponds to totally geodesic points of the polar map.

Example 4.6. R.L. Bryant studied in [3] an interesting class of branched minimal surfaces in $\mathbb{S}^{4}$, the so called superminimal ones. Actually, a branched minimal surface is called superminimal if at each point the ellipse of curvature is a circle or a point. Moreover, Bryant has shown that all superminimal surfaces in $\mathbb{S}^{4}$ admit a "Weierstrass type representation" in terms of meromorphic functions. More precisely, let $\mathbb{C P}^{3}$ be the complex projective space endowed with the Fubini-Study metric and $\mathcal{P}: \mathbb{C P}^{3} \rightarrow \mathbb{S}^{4}$ the Penrose twistor fibration. Consider two meromorphic functions $\phi$ and $\psi$ defined on a Riemannian surface $M^{2}$ and let $G: M^{2} \rightarrow \mathbb{C P}^{3}$ be the map given by

$$
G(z):=\left[1: \phi-\frac{1}{2} \psi \frac{d \phi}{d \psi}: \psi: \frac{1}{2} \frac{d \phi}{d \psi}\right] .
$$

Then $G$ is holomorphic, horizontal with respect to the Penrose fibration and $\mathcal{P} \circ G$ gives rise to a superminimal surface in $\mathbb{S}^{4}$. Conversely, any superminimal surface in 
$\mathbb{S}^{4}$ can be produced, at least locally, in this way. Applying the polar map over superminimal surfaces we can produce examples of minimal hypersurfaces (even compact ones) in $\mathbb{S}^{4}$ with vanishing Gauss-Kronecker curvature. For example, consider the pair of meromorphic functions $\phi, \psi: \mathbb{C P}^{1}=\mathbb{C} \cup\{\infty\} \rightarrow \mathbb{C}$, given by $\phi(z)=z^{5}$ and $\psi(z)=z^{2}$. Composing the holomorphic curve $G: \mathbb{C P}^{1} \rightarrow \mathbb{C P}^{3}$,

$$
G(z)=\left[1:-\frac{1}{4} z^{5}: z^{2}: \frac{5}{4} z^{3}\right]
$$

with the Penrose fibration, we obtain a superminimal surface having at $z=0$ and $z=\infty$ branch points of order 1 . Moreover, it turns out that the polar map over $\mathcal{P} \circ G$ is everywhere regular and the points of the fibers over $z_{1}=0$ and $z_{2}=\infty$ corresponds to totally geodesic points of the polar map.

Consider the hyperbolic space $\mathbb{H}^{4}$ as the hyperquadric

$$
\mathbb{H}^{4}:=\left\{x=\left(x_{1}, x_{2}, x_{3}, x_{4}, x_{5}\right) \in \mathbb{R}_{1}^{5}:\langle x, x\rangle_{1}=-1, \quad x_{1}>0\right\} .
$$

The hyperquadric

$$
\mathbb{S}_{1}^{4}:=\left\{x \in \mathbb{R}_{1}^{5}:\langle x, x\rangle_{1}=1\right\},
$$

is called the de Sitter space. Let $M^{2}$ be an oriented manifold of dimension 2 and $g: M^{2} \rightarrow \mathbb{S}_{1}^{4} \subset \mathbb{R}_{1}^{5}$ a branched spacelike stationary immersion. The timelike unit normal bundle $\mathcal{N}_{-1}(g)$ of $g$, defined by

$$
\mathcal{N}_{-1}(g):=\left\{(x, w) \in M^{2} \times \mathbb{R}_{1}^{5}:\langle w, w\rangle_{1}=-1, w \perp \mathbb{R} \cdot g+d g\left(T_{x} M^{2}\right)\right\}
$$

is a smooth manifold of dimension 3 and the polar map $\Psi_{g}: \mathcal{N}_{-1}(g) \rightarrow \mathbb{H}^{4}$ is defined by the formula

$$
\Psi_{g}(x, w):=w .
$$

Proceeding in the same way as in Proposition 4.4, we can prove the following result.

Proposition 4.7. Let $g: B \rightarrow \mathbb{S}_{1}^{4} \subset \mathbb{R}_{1}^{5}$ be a branched spacelike stationary surface, having $z=0$ as a branch point of order $m$ and being everywhere else regular. Then,

a) a point $(z, w), z \neq 0$, is a regular point of $\Psi_{g}$ if and only if the shape operator $A_{w}$ of $g$, with respect to the normal $w$, is non-singular. Moreover, $\Psi_{g}$ is regular at a point of the form $(0, w)$, if and only if there exists a negative number $l$ such that

$$
\lim _{z \rightarrow 0}|z|^{2 m} \operatorname{det} A_{w}=l<0 .
$$

b) On the open set of its regular points, the polar map is minimal with vanishing Gauss-Kronecker curvature.

Example 4.8. Surfaces in $\mathbb{S}^{3}$ which are critical points of the functional

$$
\mathcal{W}\left(M^{2}\right)=\int_{M^{2}}\left(H^{2}+1-K\right) d M
$$

where $H, K$ are the mean and Gaussian curvature of the surface $M^{2}$, are called Willmore surfaces. Bryant showed in [4 that there is a duality between branched spacelike stationary surfaces in $\mathbb{S}_{1}^{4}$ and Willmore surfaces in $\mathbb{S}^{3}$. More precisely, let $h: M^{2} \rightarrow \mathbb{S}^{3} \subset \mathbb{S}_{1}^{4}$ be an isometric immersion of a 2-dimensional oriented Riemannian manifold $M^{2}$. Consider $\mathbb{S}^{3}$ as the submanifold of $\mathbb{S}_{1}^{4}$

$$
\mathbb{S}^{3}=\left\{(0, y) \in \mathbb{R}_{1}^{5}=\mathbb{R}_{1}^{1} \times \mathbb{R}^{4}:\langle y, y\rangle_{1}=1\right\},
$$


and define the conformal Gauss map $\mathcal{G}_{h}: M^{2} \rightarrow \mathbb{S}_{1}^{4}$,

$$
\mathcal{G}_{h}(x):=H(x)(1, h(x))+(0, \eta(x)), \quad x \in M^{2},
$$

where $\eta$ is a unit normal vector field along $h$. The induced by $\mathcal{G}_{h}$ metric tensor $\langle,\rangle_{\sim}$ on $M^{2}$ is related with the Riemannian metric $\langle$,$\rangle of M^{2}$ by the formula

$$
\langle,\rangle_{\sim}=\left(H^{2}+1-K\right)\langle,\rangle,
$$

where $K$ stands for the Gaussian curvature and $H$ for the mean curvature of $h$. We point out that the zeroes of $H^{2}+1-K$ are isolated, unless $g$ is totally umbilical. Moreover, $\mathcal{G}_{h}$ is a branched spacelike stationary surface if and only if h is a Willmore surface. In fact, branched spacelike stationary surfaces in $\mathbb{S}_{1}^{4}$ arise as conformal Gauss maps of a Willmore surfaces in $\mathbb{S}^{3}$. Minimal surfaces in $\mathbb{S}^{3}$ are the simplest examples of Willmore surfaces.

Example 4.9. Examples of complete minimal hypersurfaces in $\mathbb{H}^{4}$ arise as hyperbolic cylinders over complete minimal surfaces in horospheres or equidistant hypersurfaces. More precisely, let $Q^{3}$ be a horosphere or equidistant hypersurface in $\mathbb{H}^{4}$ and denote by $\eta$ a unit normal vector field of $Q^{3}$ in $\mathbb{H}^{4}$. If $h: M^{2} \rightarrow Q^{3} \subset \mathbb{H}^{4}$ is a complete minimal surface, then the hyperbolic cylinder $F_{h}: M^{2} \times \mathbb{R} \rightarrow \mathbb{H}^{4}$, given by

$$
F_{h}(z, t):=\cosh t h(z)+\sinh t \eta(z), \quad(z, t) \in M^{2} \times \mathbb{R}
$$

provides a complete minimal hypersurface in $\mathbb{H}^{4}$ with vanishing Gauss-Kronecker curvature. In fact, these are the only known examples of complete minimal hypersurface in $\mathbb{H}^{4}$ with vanishing Gauss-Kronecker curvature.

\section{The Nullity Distribution}

Let $M^{3}$ be a 3-dimensional, oriented Riemannian manifold and $f: M^{3} \rightarrow \mathbb{Q}_{c}^{4}$ an isometric immersion. Denote by $\xi$ a unit normal vector field along $f$ and by $A$ its shape operator. From the Weingarten formula it follows that

$$
d \xi=-d f \circ A \text {. }
$$

The eigenvalues

$$
k_{1} \geq k_{2} \geq k_{3}
$$

of $A$ are called principal curvatures of the immersion. Recall that the mean curvature $H$, the square $S$ of the second fundamental form and the Gauss-Kronecker curvature $K$ of the immersion are given in terms of the principal curvatures by the following formulas

$$
H=\frac{1}{3}\left(k_{1}+k_{2}+k_{3}\right), \quad S=k_{1}^{2}+k_{2}^{2}+k_{3}^{2}, \quad K=k_{1} k_{2} k_{3} .
$$

Let us assume that $f$ is minimal and that the type number of the immersion is at most 2. Equivalently, $f$ is minimal with Gauss-Kronecker curvature identically zero. A point $x_{0}$ where the square $S$ of the second fundamental form vanishes is called a totally geodesic point.

Assume at first that $x$ is a non-totally geodesic point of $f$. Then, there is a simply connected neighborhood $\Omega$ around $x$, where the second fundamental form of $f$ is nowhere zero. Thus, the principal curvatures of $f$ on $\Omega$ are $k_{1}=\lambda, k_{2}=0$, $k_{3}=-\lambda$, where $\lambda$ is a smooth positive function on $\Omega$. Moreover, we can choose on $\Omega$ an orthonormal frame field $\left\{e_{1}, e_{2}, e_{3}\right\}$ of principal directions corresponding to $\lambda$, $0,-\lambda$, respectively. Let $\left\{\omega_{1}, \omega_{2}, \omega_{3}\right\}$ and $\left\{\omega_{i j}\right\}, i, j \in\{1,2,3\}$, be the dual coframe 
and the connection forms, respectively. Define the functions $u, v: U \rightarrow \mathbb{R}$, given by the formulas

$$
u=\left\langle\nabla_{e_{3}} e_{1}, e_{2}\right\rangle \text { and } v=\left\langle\nabla_{e_{1}} e_{1}, e_{2}\right\rangle .
$$

From the Gauss and Codazzi equations, follows that

$$
\begin{aligned}
& \omega_{12}\left(e_{1}\right)=v, \quad \omega_{13}\left(e_{1}\right)=\frac{1}{2} e_{3}(\log \lambda), \quad \omega_{23}\left(e_{1}\right)=u, \\
& \omega_{12}\left(e_{2}\right)=0, \quad \omega_{13}\left(e_{2}\right)=\frac{1}{2} u, \quad \omega_{23}\left(e_{2}\right)=0, \\
& \omega_{12}\left(e_{3}\right)=u, \quad \omega_{13}\left(e_{3}\right)=-\frac{1}{2} e_{1}(\log \lambda), \quad \omega_{23}\left(e_{3}\right)=-v,
\end{aligned}
$$

and

$$
\begin{array}{ll}
e_{2}(v)=v^{2}-u^{2}+c, & e_{1}(u)=e_{3}(v), \\
e_{2}(u)=2 u v, & e_{3}(u)=-e_{1}(v) .
\end{array}
$$

The above equations yield

$$
\begin{aligned}
& {\left[e_{1}, e_{2}\right]=-v e_{1}+\frac{1}{2} u e_{3},\left[e_{2}, e_{3}\right]=\frac{1}{2} u e_{1}+v e_{3},} \\
& {\left[e_{1}, e_{3}\right]=-\frac{1}{2} e_{3}(\log \lambda) e_{1}-2 u e_{2}+\frac{1}{2} e_{1}(\log \lambda) e_{3} .}
\end{aligned}
$$

The following observation is a fact of basic importance.

Lemma 5.1. The functions $u$ and $v$ are harmonic.

Proof. From the equations (5.1) and the definition of the Laplacian, we obtain

$$
\begin{aligned}
\Delta u= & e_{1} e_{1}(u)+e_{2} e_{2}(u)+e_{3} e_{3}(u) \\
& -\nabla_{e_{1}} e_{1}(u)-\nabla_{e_{2}} e_{2}(u)-\nabla_{e_{3}} e_{3}(u) \\
= & e_{1} e_{1}(u)+e_{2} e_{2}(u)+e_{3} e_{3}(u) \\
& -2 v e_{2}(u)-\frac{1}{2} e_{1}(\log \lambda) e_{1}(u)-\frac{1}{2} e_{3}(\log \lambda) e_{3}(u) .
\end{aligned}
$$

Moreover, in view of (5.2), we get

$$
e_{1} e_{1}(u)=e_{1} e_{3}(v), e_{3} e_{3}(u)=-e_{3} e_{1}(v), e_{2} e_{2}(u)=2 v e_{2}(u)+2 u e_{2}(v) .
$$

Combining (5.2), (5.3) and the above mentioned relations, we obtain $\Delta u=0$. Similarly we verify that $v$ is harmonic.

In the sequel, based on ideas developed by Ramanathan [20, we intend to give a careful analysis of the set of totally geodesic points

$$
\mathcal{A}:=\left\{x \in M^{3}: S(x)=0\right\} .
$$

Since $f$ is a minimal immersion, it follows that $f$ is real analytic. Hence, the square $S$ of the length of the second fundamental form is a real analytic function and the set $\mathcal{A}$ is real analytic. According to Lojasewicz's Structure Theorem 14, Theorem 6.3 .3 , p. 168], $\mathcal{A}$ can be locally decomposed as

$$
\mathcal{A}=\mathcal{V}^{0} \cup \mathcal{V}^{1} \cup \mathcal{V}^{2} \cup \mathcal{V}^{3}
$$

where $\mathcal{V}^{m}, 0 \leq m \leq 3$, is either empty or a finite, disjoint union of $m$-dimensional subvarieties. A point $x_{0} \in \mathcal{A}$ is called smooth point of dimension $m$, if there is a neighborhood $\Omega$ of $x_{0}$ such that $\Omega \cap \mathcal{A}$ is an $m$-dimensional real analytic submanifold of $\Omega$. Otherwise, the point $x_{0}$ is said singular.

The distribution

$$
p \in M^{n} \rightarrow \mathcal{D}(p):=\operatorname{ker} A(p)
$$


is called the nullity distribution of $f$. From our assumptions it follows that if $x$ is a point of $M^{n}$ then, either $\operatorname{dim} \mathcal{D}(x)=1$ or $\operatorname{dim} \mathcal{D}(x)=3$. Obviously, the dimension of the nullity distribution is 3 precisely at the totally geodesic points of $M^{3}$.

Lemma 5.2. Let $\Omega$ be an open subset of $M^{3}$ where the dimension of the nullity distribution is constant. Then $\mathcal{D}$ is differentiable and involutive. The leaves of $\mathcal{D}$ are totally geodesic in $M^{3}$ and their images through $f$ are totally geodesic submanifolds in $\mathbb{Q}_{c}^{4}$. Moreover, the Gauss map of $f$ is constant along the leaves of $\mathcal{D}$.

Proof. Assume at first that the dimension of the nullity distribution in $\Omega$ is 3 . Then all the points of $\Omega$ are totally geodesic and consequently, the image of $\Omega$ through $f$ lies in a totally geodesic hypersurface of $\mathbb{Q}_{c}^{4}$. Suppose now that the dimension of $\mathcal{D}$ in $\Omega$ is 1 . Let $\gamma:(-\varepsilon, \varepsilon) \rightarrow \Omega$ be an integral curve of the vector field $e_{2}$. From relations (5.1) it follows that

$$
\nabla_{e_{2}} e_{2}=\omega_{21}\left(e_{2}\right) e_{1}+\omega_{23}\left(e_{2}\right) e_{3}=0 .
$$

Thus, $\gamma$ is geodesic in $M^{3}$. Furthermore, from the Gauss formula we obtain

$$
\nabla_{e_{2}} d f\left(e_{2}\right)=d f\left(\nabla_{e_{2}} e_{2}\right)+\left\langle A e_{2}, e_{2}\right\rangle \xi=0
$$

and thus, the curve $f \circ \gamma:(-\varepsilon, \varepsilon) \rightarrow \mathbb{Q}_{c}^{4}$ is a geodesic. Finally, from the Weingarten formula $d \xi=-d f \circ A$, we deduce that the Gauss map of $f$ is constant along the leaves of $\mathcal{D}$.

The following important result is due of D. Ferus [10, Lemma 2, p. 311].

Lemma 5.3. Let $\gamma:[0, b] \rightarrow M^{3}$ be a geodesic curve such that $\gamma \prime(t) \in \mathcal{D}$, for any $t \in[0, b)$, and $\operatorname{dim} \mathcal{D}(\gamma(t))=m$, for any $t \in[0, b)$. Then, $\operatorname{dim} \mathcal{D}(\gamma(b))=m$.

Proof. Set $E(t)=\gamma^{\prime}(t)=e_{2} \circ \gamma(t), t \in[0, b)$, and consider a vector field $Y$ along $\gamma:[0, b) \rightarrow M^{3}$ such that

$$
\left\{\begin{array}{l}
Y(0) \perp E(0), \\
{[Y, E]=0 .}
\end{array}\right.
$$

The existence and uniqueness of $Y$ are immediate since this is a Cauchy problem for an ordinary linear equation of first order. Note that from (5.4), we obtain

$$
\frac{d Y}{d t}=\nabla_{E} Y=\nabla_{Y} E
$$

Furthermore, we claim that $Y$ is normal along $\gamma:[0, b) \rightarrow M^{3}$. Indeed,

$$
\frac{d}{d t}\langle E, Y\rangle=\left\langle\nabla_{E} E, Y\right\rangle+\left\langle E, \nabla_{Y} E\right\rangle=\left\langle E, \nabla_{Y} E\right\rangle=\frac{1}{2} Y\langle E, E\rangle=0 .
$$

Because $\langle E(0), Y(0)\rangle=0$, it follows that $\langle E(t), Y(t)\rangle=0$, for any $t \in[0, b)$. Differentiating the relation (5.5), we deduce that

$$
\frac{d^{2} Y}{d t^{2}}=\nabla_{E} \nabla_{Y} E
$$

On the other hand we have

$$
R(E, Y) E=\nabla_{E} \nabla_{Y} E-\nabla_{Y} \nabla_{E} E-\nabla_{[E, Y]} E=\nabla_{E} \nabla_{Y} E=\frac{d^{2} Y}{d t^{2}}
$$


where $R$ stands for the Riemannian curvature tensor of $M^{3}$. Moreover, the Gauss equation implies that

$$
R(E, Y) E=c\{\langle Y, E\rangle E-Y\}=-c Y .
$$

Thus, combining the equations (5.6) and (5.7), we obtain

$$
\frac{d^{2} Y}{d t^{2}}+c Y=0
$$

This is a second order ordinary linear differential equation with constant coefficients in $[0, b)$ and thus, the solution can be extended for $t=b$. Consider now a parallel vector field $Z$ along $\gamma:[0, b] \rightarrow M^{3}$ and let $G:[0, b] \rightarrow \mathbb{R}$ the function given by

$$
G(t):=\langle A Y(t), Z(t)\rangle, \quad t \in[0, b] .
$$

Differentiating, we obtain

$$
\begin{aligned}
\frac{d G}{d t} & =E\langle A Y, Z\rangle=\left\langle\nabla_{E} A Y, Z\right\rangle=\left\langle\left(\nabla_{E} A\right) Y+A \nabla_{E} Y, Z\right\rangle \\
& =\left\langle\left(\nabla_{Y} A\right) E+A \nabla_{E} Y, Z\right\rangle=\left\langle-A \nabla_{Y} E+A \nabla_{E} Y, Z\right\rangle \\
& =\langle A[E, Y], Z\rangle \\
& =0 .
\end{aligned}
$$

Thus, $G$ is constant along $\gamma$. Taking $Z(0)=Y(0)$, we get that

$$
\langle A Y, Z\rangle(\gamma(b))=\langle A Y, Z\rangle(\gamma(0)) .
$$

Therefore,

$$
\operatorname{dim} \mathcal{D}(\gamma(0))=\operatorname{dim} \mathcal{D}(\gamma(b))
$$

and the proof is completed.

Denote by $\mathcal{F}$ the foliation of the nullity distribution in the open subset $M^{3}-\mathcal{A}$. According to Lemma 5.2, the foliation consists of geodesics of $M^{3}$. Our aim is to prove that $\mathcal{F}$ can be analytically extended over the set $\mathcal{A}$. In the following we shall prove that $\mathcal{A}$ contains only smooth points of dimension 1.

Lemma 5.4. There are no smooth points of dimension 3 in $\mathcal{A}$, unless $f$ is totally geodesic.

Proof. Suppose that $x_{0}$ is a smooth point of $\mathcal{A}$ with dimension 3. Then, there exists an open neighborhood $\Omega$ of $M^{3}$ such that $x_{0} \in \Omega \subset \mathcal{A}$. From Lemma 5.2, it follows that $f: \Omega \rightarrow \mathbb{Q}_{c}^{4}$ is totally geodesic and hence, according to the Unique Continuation Property, $f: M^{3} \rightarrow \mathbb{Q}_{c}^{4}$ must be totally geodesic.

Lemma 5.5. There are no smooth points of dimension 2 in $\mathcal{A}$.

Proof. Suppose in contrary that $x_{0}$ is a smooth point of $\mathcal{A}$ with dimension 2. Then there exists an open neighborhood $\Omega$ of $M^{3}$ around $x_{0}$ such that $L^{2}=\Omega \cap \mathcal{A}$ is a 2-dimensional imbedded surface. Consider the function $h: M^{3} \rightarrow \mathbb{R}$, given by

$$
h:= \begin{cases}\langle\xi, \alpha\rangle, & \text { if } \quad c=0 \\ \left\langle\xi, f\left(x_{0}\right)\right\rangle, & \text { if } \quad c \in\{-1,1\}\end{cases}
$$

where $\alpha$ is a vector of $\mathbb{R}^{4}$ such that $h\left(x_{0}\right)=0$. A simple calculation implies,

$$
\Delta h+S h=0,
$$


where $\Delta$ is the Laplacian of $M^{3}$. On the other hand, for any $x \in L^{2}$ and $X \in T_{x} M^{3}$ it holds

$$
d h(X)=0 \text { and } h(x)=0 .
$$

We claim that $h$ is identically zero. Indeed, let $(z ; t) \in L^{2} \times(-\varepsilon, \varepsilon)$ real analytic coordinates centered at $x_{0}$. Then, $h$ has a representation of the form,

$$
h(z ; t)=t^{n} h_{0}(z ; t),
$$

where $h_{0}: \Omega \rightarrow \mathbb{R}$, is a non vanishing real analytic function. Let $z_{1} \in L^{2}$ be a point where $h_{0}\left(z_{1} ; 0\right) \neq 0$. By continuity there is an $r>0$, such that $h_{0}$ is not zero in the set

$$
V=B_{\varepsilon}\left(z_{1} ; 0\right) \cap\left(L^{2} \times(0, \varepsilon)\right) .
$$

where $B_{\varepsilon}\left(z_{1} ; 0\right) \subset \Omega$ is the open ball of radius $r$ centered at $\left(z_{1}, 0\right)$. Thus, the restriction of the function $h$ at $V$ has a fixed sign. Assume at first that $h$ is positive in $V$. Then $\Delta h \leq 0$ on $V$. Also note that $h$ attains a relative minimum at the boundary point $\left(z_{1} ; 0\right)$. Consequently, by the Hopf Boundary Point Lemma the outward normal derivative of $h$ at $\left(z_{1} ; 0\right)$ must be non-zero. This contradicts with the fact that the differential of $h$ vanishes identically at $\left(z_{1}, 0\right)$. The same contradiction occurs if we assume that $h$ is negative in $V$. Thus, $h$ must be identically zero on $\Omega$, which implies that $f: \Omega \rightarrow \mathbb{Q}_{c}^{4}$ is totally geodesic. Hence, all the points of $\Omega$ are smooth with dimension 3 which contradicts to our assumption. Therefore, there are no smooth points of $\mathcal{A}$ with dimension 2 .

Lemma 5.6. The set $\mathcal{A}$ does not contain any isolated point.

Proof. Suppose in contrary that $x_{0}$ is an isolated point of $\mathcal{A}$. Then, there exists an open neighborhood $\Omega$ of $M^{3}$ around $x_{0}$, such that

$$
\operatorname{dim} \mathcal{D}(x)=\left\{\begin{array}{lll}
1, & \text { if } & x \in \Omega-\left\{x_{0}\right\} \\
3, & \text { if } \quad x=x_{0}
\end{array}\right.
$$

Let $\left\{x_{n}\right\}$ be a sequence of points of $\Omega-\left\{x_{0}\right\}$ converging to $x_{0}$ and $E_{n}=e_{2}\left(x_{n}\right) \in$ $T_{x_{n}} M^{3}$ the sequence of unit vectors contained in the nullity distribution of $f$. By passing to a subsequence, if necessary, we may assume that there is a vector $E_{0} \in$ $T_{x_{0}} M^{3}$ such that $\lim _{n \rightarrow \infty} E_{n}=E_{0}$. Consider now the geodesic flow $\Phi:(-\varepsilon, \varepsilon) \times \Sigma \rightarrow$ $\Omega$, where

$$
\Sigma:=\left\{(x, E(x)) \in T_{x} \Omega:|E(x)|=1\right\} .
$$

For a fixed pair $\left(x, e_{2}(x)\right)$, the curve $\gamma:(-\varepsilon, \varepsilon) \rightarrow \Omega$ given by

$$
\gamma(t):=\Phi\left(t, x, e_{2}(x)\right),
$$

is the geodesic passing through $x$ with tangent vector $e_{2} \in T_{x} M^{3}$. By continuity, we deduce that

$$
\lim _{n \rightarrow \infty} \Phi\left(t, x_{n}, E_{n}\right)=\Phi\left(t, x_{0}, E_{0}\right) \text { and } \lim _{n \rightarrow \infty} \frac{\partial \Phi}{\partial t}\left(t, x_{n}, E_{n}\right)=\frac{\partial \Phi}{\partial t}\left(t, x_{0}, E_{0}\right),
$$

where now $\gamma_{0}(t):=\Phi\left(t, x_{0}, E_{0}\right)$ is the geodesic passing through $x_{0}$ with tangent vector $E_{0} \in T_{x_{0}} M^{3}$. Moreover, since $\left\|A \gamma_{n}^{\prime}(t)\right\|=0$, again by continuity, we obtain that $\left\|A \gamma_{0}^{\prime}(t)\right\|=0$, for any $t \in(-\varepsilon, \varepsilon)$. So, $\gamma_{n}^{\prime}(t) \in \mathcal{D}$ for any $t \in(-\varepsilon, \varepsilon)$. Observe now that

$$
\operatorname{dim} \mathcal{D}(\gamma(t))= \begin{cases}1, & t \neq 0 \\ 3, & t=0\end{cases}
$$

This contradicts Lemma 5.3, thus such a point $x_{0}$ does not exist. 
Lemma 5.7. The foliation $\mathcal{F}$ of the nullity distribution can be extended continuously over the smooth points of $\mathcal{A}$.

Proof. Let $\Omega$ be an open subset of $M^{3}$ such that $\Omega \cap \mathcal{A}$ is a smooth embedded curve. Consider, as in Lemma 5.6, a sequence of points $\left\{x_{n}\right\} \in \Omega-\mathcal{A}$ and a sequence of unit vectors $E_{n} \in \mathcal{D}$ such that $\lim _{n \rightarrow \infty} x_{n}=x_{0} \in \mathcal{A}$ and $\lim _{n \rightarrow \infty} E_{n}=E_{0} \in T_{x_{0}} M^{3}$. Using again the geodesic flow $\Phi$, we deduce that the tangent vector $\gamma_{0}^{\prime}(t)$ of the geodesic curve

$$
\gamma_{0}(t):=\lim _{n \rightarrow \infty} \Phi\left(t, x_{n}, E_{n}\right),
$$

belongs to $\mathcal{D}$, for any $t \in(-\varepsilon, \varepsilon)$. By virtue of Lemma $5.3, \gamma_{0}$ must lie entirely in $\mathcal{A}$. If we consider another sequence $\left\{\widetilde{x}_{n}\right\}$ tending at $x_{0}$ and another sequence of vectors $\widetilde{E}_{n}$ tending at $\widetilde{E}_{0}$, then repeating the same argument as above we deduce that the limit line must lie in $\mathcal{A}$ and so $E_{0}= \pm \widetilde{E}_{0}$. Hence, there is a continuous extension of the line field $\mathcal{F}$ to the set $\mathcal{A}$.

Lemma 5.8. The foliation $\mathcal{F}$ of the nullity distribution can be extended analytically over the smooth points of $\mathcal{A}$.

Proof. Let $T M^{3}$ be the tangent bundle of the Riemannian manifold $M^{3}$ and endow it with the Sasaki metric $\langle,\rangle_{\sim}$. In fact, if $X$ and $Y$ are tangent vectors at the time $t=0$ to the curves $\widetilde{\alpha}(t)=(\alpha(t), V(t)), \widetilde{\beta}(t)=(\beta(t), W(t))$ on $T M^{3}$, then

$$
\langle X, Y\rangle_{\sim}:=\left\langle\alpha^{\prime}(0), \beta^{\prime}(0)\right\rangle+\left\langle V_{t}(0), W_{t}(0)\right\rangle
$$

where $V_{t}$ and $W_{t}$ stand for the covariant derivatives of the vector fields $V, W$ along the curves $\alpha(t), \beta(t)$ on $M^{3}$. Denote by $T_{1} M^{3}$ the unit tangent bundle of $T M^{3}$. Here $T_{1} M^{3}$ is regarded as a submanifold of $T M^{3}$ equipped with the induced Riemannian metric by the Sasaki metric of $T M^{3}$. Assume now that $\Omega$ is an open subset of $M^{3}-\mathcal{A}$ and that $\left\{e_{1}, e_{2}, e_{3}\right\}$ is a local frame of principal directions on $\Omega$, such that

$$
A e_{1}=\lambda e_{1}, A e_{2}=0, A e_{3}=-\lambda e_{3} .
$$

Define the smooth map $F: \Omega \rightarrow T_{1} M^{3}$, by

$$
F(x):=\left(x, e_{2}(x)\right), \quad x \in \Omega .
$$

We claim that the map $F$ is harmonic. Indeed, from the relations (5.1) and (5.2) we can compute the rough Laplacian $\widetilde{\Delta} F$ of $F$,

$$
\begin{aligned}
\widetilde{\Delta} F & =\sum_{i=1}^{3}\left\{\nabla_{e_{i}} \nabla_{e_{i}} F-\nabla_{\nabla_{e_{i}} e_{i}} F\right\} \\
& =\nabla_{e_{1}} \nabla_{e_{1}} e_{2}+\nabla_{e_{3}} \nabla_{e_{3} e_{2}}-\nabla_{\nabla_{e_{1}} e_{1}} e_{2}-\nabla_{\nabla_{e_{1}} e_{1}} e_{2} \\
& =-2\left(u^{2}+v^{2}\right) F \\
& =-2\left(\left\|\nabla_{e_{1}} F\right\|^{2}+\left\|\nabla_{e_{3}} F\right\|^{2}\right) F .
\end{aligned}
$$

Hence, the map $F$ satisfies the differential equation

$$
\widetilde{\Delta} F+2\|\nabla F\|^{2} F=0,
$$

which is precisely the Euler-Lagrange equation for the energy of the map $F$ (cf., 23. Proposition 1.1]). Consequently, $F$ is harmonic. Moreover, according to Lemma 5.7, the map $F$ can be extended continuously on $\mathcal{A}$. Consequently, from Theorem 2.2 , we deduce that $F$ can be extended analytically over $\mathcal{A}$. Hence, the foliation $\mathcal{F}$ 
of the nullity distribution can be extended analytically over the smooth points of $\mathcal{A}$.

Lemma 5.9. There are no singular points on the set $\mathcal{A}$.

Proof. Assume in contrary that $x_{0}$ is a singular point of $\mathcal{A}$. From Lemmata 5.4, 5.5 and 5.6, it follows that $\mathcal{A}$ contains algebraic curves. The singular points of an algebraic curve are either cusps, intersection points or combination of those. Furthermore, the singular points are isolated. Suppose at first that $x_{0}$ is an intersection point. Let $\Omega$ be an open neighborhood around $x_{0}$, such that the restriction of $f$ on $\Omega$ is injective, and consider a transversal $L^{2}$ to the foliation $\mathcal{F}$ on $U-\left\{x_{0}\right\}$. Since the images of the integral curves of $\mathcal{D}$ via $f$ are geodesics lines in $\mathbb{Q}_{c}^{4}$, the map $f$ can be represented by the formula,

$$
f(z, t)= \begin{cases}\cos t f(z, 0)+\sin t d f\left(e_{2}\right)(z, 0), & c=1, \\ f(z, 0)+t d f\left(e_{2}\right)(z, 0), & c=0, \\ \cosh t f(z, 0)+\sinh t d f\left(e_{2}\right)(z, 0), & c=-1 .\end{cases}
$$

where $z \in L^{2}$. From the above description, it follows that there are points $\left(z_{1}, t_{1}\right)$ and $\left(z_{2}, t_{2}\right)$ such that $f\left(z_{1}, t_{1}\right)=f\left(x_{0}\right)=f\left(z_{2}, t_{2}\right)$, which is a contradiction. Suppose now that $x_{0}$ is a cusp. Since the images of the leaves of the nullity foliation via $f$ are geodesics in $\mathbb{Q}_{c}^{4}$, we deduce that there must be a point $x_{1} \in \Omega, x_{1} \neq x_{0}$, where the leaves of $\mathcal{F}$ intersect. This leads again to a contradiction. Thus, there are no singular points on $\mathcal{A}$.

\section{Proofs of theorems}

Proof of Theorem 1.1. Assume that $f$ is not totally geodesic. It is enough to examine only the case where the set $\mathcal{A}$ of the totally geodesic points of $f$ is non empty. By virtue of the results in Section 5, the set $\mathcal{A}$ contains geodesic lines and the foliation $\mathcal{F}$ of the nullity distribution $\mathcal{D}$ is well defined and regular in the sense of Palais 19. Consider a point $x_{0} \in \mathcal{A}$ and a coordinate system $\left(x_{1}, x_{2}, x_{3}\right)$ on $\Omega \subset M^{3}$, around $x_{0}$, such that $\partial / \partial x_{2}=e_{2}$. Denote by $M^{2}=\Omega / \mathcal{F}$ the space of leaves of $\mathcal{F}$ on $\Omega$ and by $\pi: \Omega \rightarrow M^{2}$ the quotient projection. It is well known that $M^{2}$ can be equipped with a differentiable structure making $\pi$ a submersion. Endow $M^{2}$ with the induced conformal structure by the Riemannian metric $\langle,\rangle_{0}$ which makes $\pi$ a Riemannian submersion.

Euclidean Case: Since the unit normal $\xi$ of the hypersurface remains constant along each leaf of $\mathcal{F}$, we may define a smooth map $g: M^{2} \rightarrow \mathbb{S}^{3}$ so that $g \circ \pi:=\xi$. Because $\xi$ is harmonic and $\pi$ is a Riemannian submersion, it follows that $g$ is also harmonic. Consider a smooth transversal $L^{2}$ to the leaves of $\mathcal{F}$ passing through the point $x_{0}$. Using the Weingarten formula, we deduce that the induced by $g$ metric tensor $\langle,\rangle_{g}$ on $M^{2}$ is given by

$$
\left.\langle,\rangle_{g} \circ \pi\right|_{L}=\left.\left.\lambda^{2}\right|_{L}\langle,\rangle_{0} \circ \pi\right|_{L}
$$

where $\left.\pi\right|_{L}$ and $\left.\lambda^{2}\right|_{L}$ is the restriction of $\pi$ and $\lambda^{2}$ to the transversal $L^{2}$, respectively. Consequently, $\pi\left(x_{0}\right)$ is a branch point of $g$ and thus $g$ is a branched minimal surface. Moreover, the normal bundle of $g$ is spanned by the vector field $\eta$ given by $\eta \circ \pi:=$ $d f\left(e_{2}\right)$. Denote by $\sigma: \Omega \rightarrow \mathbb{R}, \sigma:=\langle\xi, f\rangle$, the support function of $f$. Because $e_{2}(\sigma)=0$, it follows that $\sigma$ is constant along the leaves of the foliation $\mathcal{F}$. Thus, the function $\gamma: M^{2} \rightarrow \mathbb{R}$ given by $\gamma \circ \pi:=\sigma$ is well defined and smooth. We may 
choose the transversal $L^{2}$ in such a way that the frame $\left\{E_{1}:=e_{1}(x), E_{3}:=e_{3}(x)\right\}$ spans $T_{x} L^{2}$, at a point $x \neq x_{0}$. Then, $\left\{X_{1}:=\frac{1}{\lambda(x)} d \pi\left(E_{1}\right), X_{3}:=\frac{1}{\lambda(x)} d \pi\left(E_{3}\right)\right\}$ constitute an orthonormal base at $T_{\pi(x)} M^{2}$ with respect to $\langle,\rangle_{g}$ and

$$
\begin{aligned}
d g(\nabla \gamma) \circ \pi(x) & =d g\left\{\left(X_{1} \gamma\right) X_{1}+\left(X_{2} \gamma\right) X_{2}\right\} \\
& =\left\langle f(x), d f\left(E_{1}\right)\right\rangle d f\left(E_{1}\right)+\left\langle f(x), d f\left(E_{3}\right)\right\rangle d f\left(E_{3}\right) \\
& =f^{\top}(x),
\end{aligned}
$$

where $f^{\top}$ stands for the tangential component of $f$ on $d f\left(T_{x} M^{3}\right)$. However, the right hand side of the above equality is well defined for any $x \in \Omega$ which implies that $d g(\nabla \gamma)$ is well defined even on the branch point. Analyzing $f$ with respect to the orthonormal basis $\left\{d f\left(e_{1}\right), d f\left(e_{2}\right), d f\left(e_{3}\right), \xi\right\}$, we deduce that

$$
f=\gamma(g \circ \pi)+d g(\nabla \gamma) \circ \pi+\left\langle f, d f\left(e_{2}\right)\right\rangle d f\left(e_{2}\right) .
$$

Moreover, $f=\Psi_{\gamma, g} \circ T$ on $\Omega$, where $T: \Omega \rightarrow \mathcal{N}(g)$ is the map defined by

$$
T(x)=\left(\pi(x),\left\langle f(x), d f\left(e_{2}(x)\right)\right\rangle d f\left(e_{2}(x)\right)\right), \quad x \in \Omega .
$$

Spherical Case: As in the Euclidean case, the map $g: M^{2} \rightarrow \mathbb{S}^{4}$ given by $g \circ \pi:=\xi$ is well defined and harmonic. The induced metric tensor by $g\langle,\rangle_{g}$ on $M^{2}$ is given by

$$
\left.\langle,\rangle_{g} \circ \pi\right|_{L}=\left.\left.\lambda^{2}\right|_{L}\langle,\rangle_{0} \circ \pi\right|_{L}
$$

where $L^{2}$ a smooth transversal to the leaves of $\mathcal{F}$ passing through the point $x_{0}$ and $\left.\lambda^{2}\right|_{L}$ is the restriction of $\lambda^{2}$ to $L^{2}$. Hence, $g: M^{2} \rightarrow \mathbb{S}^{4}$ is a branched minimal surface. Moreover, the normal bundle of $g$ is spanned by the vector fields $\eta_{1}, \eta_{2}$ given by $\eta_{1} \circ \pi:=d f\left(e_{2}\right)$ and $\eta_{2} \circ \pi:=f$. Furthermore, $f=\Psi_{g} \circ T$ on $\Omega$, where the map $T: \Omega \rightarrow \mathcal{N}_{1}(g)$ is defined by $T(x)=(\pi(x), f(x)), x \in \Omega$.

Hyperbolic Case: The proof is the same as in the spherical case.

Proof of Theorem 1.2. The assumption in [11, 12, 13, that $f$ is free of totally geodesic points was used only to show that the local harmonic functions

$$
u=\left\langle\nabla_{e_{3}} e_{1}, e_{2}\right\rangle \text { and } v=\left\langle\nabla_{e_{1}} e_{1}, e_{2}\right\rangle
$$

can be defined globally on $M^{3}$. However, due to results in Section 5 , the local functions $u$ and $v$ can be extended analytically on $M^{3}$ even in the case when $f$ allows totally geodesic points. Indeed, suppose that $f$ is not totally geodesic. Then, the foliation $\mathcal{F}$ of the nullity distribution $\mathcal{D}$ can be extended analytically on the set $\mathcal{A}$ of the totally geodesic points of $f$. Without loss of generality we may assume that there is a global unit section $e_{2} \in \mathcal{D}$, since otherwise we can pass to the 2-fold covering space

$$
\widetilde{M^{3}}:=\left\{\left(x, \varepsilon_{x}\right): x \in M^{3}, \varepsilon_{x} \in \mathcal{D}(x)\right\} .
$$

Since the section $e_{2}$ is globally defined, the local functions $u$ and $v$ can be extended analytically to harmonic functions defined on all of $M^{3}$. Consequently, following the same arguments as in [11, 12, 13, we can complete the proof.

\section{ACKNowledgements}

The author is grateful to professor J.-H. Eschenburg for his helpful comments and suggestions. Moreover, the author would like also to thank professors A. Tertika and S. Filippa for many fruitful discussions. 


\section{REFERENCES}

[1] S.C. de Almeida and F.G.B. Brito, Minimal hypersurfaces of $\mathbb{S}^{4}$ with constant GaussKronecker curvature, Math. Z. 195 (1987), 99-107.

[2] R. Beez, Zur Theorie des Krümmungsmasses von Mannigfaltigkeiten höhere Ordnung, Zeit. für Math. und Physik 21 (1876), 373-401.

[3] R.L. Bryant, Conformal and minimal immersions of compact surfaces into the 4-sphere, J. Differential Geom. 17 (1982), 455-473.

[4] R.L. Bryant, A duality theorem for Willmore surfaces, J. Differential Geom. 20 (1984), 23-53.

[5] E. Cartan, La déformation des hypersurfaces dans l'espace euclidien réel a $n$ dimensions, Bull. Soc. Math. France 44 (1916), 65-99.

[6] J. Eells and J. H. Sampson, Harmonic mappings of Riemannian manifolds, Amer. J. Math. 86 (1964), 109-160.

[7] J.-H. Eschenburg and R. Tribuzy, Branch points of conformal mappings of surfaces, Math. Ann. 279 (1988), 621-633.

[8] L. Evans and R. Gariepy, Measure Theory and Fine Properties of Functions, Studies in Advanced Mathematics, CRC Press, Boca Raton, FL, 1992.

[9] M. Dajczer and D. Gromoll, Gauss parametrizations and rigidity aspects of submanifolds, J. Differential Geom. 22 (1985), 1-12.

[10] D. Ferus, On the type number of hypersurfaces in spaces of constant curvature, Math. Ann. 187 (1970), 310-316.

[11] T. Hasanis, A. Savas-Halilaj and T. Vlachos, Minimal hypersurfaces with zero GaussKronecker curvature, Illinois J. Math. 49 (2005), 523-529.

[12] T. Hasanis, A. Savas-Halilaj and T. Vlachos, Complete minimal hypersurfaces of $\mathbb{S}^{4}$ with zero Gauss-Kronecker curvature, Math. Proc. Cambridge Philos. Soc. 142 (2007), 125-132.

[13] T. Hasanis, A. Savas-Halilaj and T. Vlachos, Complete minimal hypersurfaces in the hyperbolic space $\mathbb{H}^{4}$ with vanishing Gauss-Kronecker curvature, Trans. Amer. Math. Soc. 359 (2007), 2799-2818.

[14] S.G. Krantz and H.R. Parks, A Primer of Real Analytic Functions, Second Edition, Birkhäuser Boston, Inc., Boston, MA, 2002.

[15] W. Killing, Die nicht-Euklidischen Raumformen in Analytische Behandlung, Teubner, Leipzig, 1885.

[16] H.B. Lawson, Complete minimal surfaces in $\mathbb{S}^{3}$, Ann. of Math. (2) 92 (1970), 335-374.

[17] M. Meier, Removable singularities of harmonic maps and an application to minimal submanifolds, Indiana Univ. Math. J. 35 (1986), 705-726.

[18] R. D. Gulliver II, R. Osserman and H.L. Royden, A theory of branched immersions of surfaces, Amer. J. Math. 95 (1973), 750-812.

[19] R. Palais, A global formulation of the Lie theory of transformation groups, Mem. Amer. Math. Soc. 22 (1957).

[20] J. Ramanathan, Minimal hypersurfaces in $\mathbb{S}^{4}$ with vanishing Gauss-Kronecker curvature, Math. Z. 205 (1990), 645-658.

[21] J.H. Sampson, Some properties and applications of harmonic mappings, Ann. Scient. de l'E.N.S, 11 (1978), 211-228.

[22] V. Sbrana, Sulla varieta ad $(n-1)$-dimensione deformabili nello spazio euclideo ad $n$ dimensione, Rend. Circ. Mat. Palermo 27 (1909), 1-45.

[23] C. M. Wood, The energy of a unit vector field, Geom. Dedicata 64 (1997), 319-330.

Department of Mathematics and Statistics, University of Cyprus, P.O. Box 20537, 1678 NiCOSIA, Cyprus

E-mail address: halilaj.andreas@ucy.ac.cy 